\section{Saúde materno-infantil e nutrição de crianças Kaiowá e Guaraní, Área Indígena de Caarapó, Mato Grosso do Sul, Brasil}

\author{
Mother-child health and nutrition of Kaiowá \\ and Guaraní indigenous children, Caarapó \\ Reserve, Mato Grosso do Sul, Brazil
}

Renata Palópoli Pícoli 1

Luana Carandina 2

Dulce Lopes Barbosa Ribas 3

\footnotetext{
1 Faculdade de Saúde Pública, Universidade de São Paulo, São Paulo, Brasil.

2 Faculdade de Medicina de Botucatu, Universidade Estadual Paulista Júlio de Mesquita Filho, Botucatu, Brasil.

3 Departamento de Tecnologia de Alimentos e Saúde Pública, Universidade Federal de Mato Grosso do Sul, Campo Grande, Brasil.

Correspondência R. P. Pícoli

Faculdade de Saúde Pública, Universidade de São Paulo. Rua Imaculada Conceição 375, Nova Andradina, MS 79750-000, Brasil. rpicoli@usp.br
}

\begin{abstract}
The objective of this study was to evaluate the nutritional status of indigenous children and to determine the conditions of mother-child health. A cross-sectional study was performed with a sample of 137 children from 0 to 59 months of age from Kaiowá and Guarani indigenous communities, Caarapó Reserve, Mato Grosso do Sul, Brazil. Interviews were carried out using a questionnaire covering the conditions of motherchild health. Nutritional evaluation was performed using anthropometric measurements (weight and height). Results showed that 19.7\% of mothers had not undergone prenatal examination and 53.3\% had home births. Malnutrition in children was $18.2 \%$ and $34.1 \%$ for the weight/age and height/age indexes, respectively. The proportion of children with malnutrition, when separated by sex, age and education level of the mother, did not show a significant statistical difference for both indexes. This study documented a high occurrence of infant malnutrition and a worrisome mother-child health situation.
\end{abstract}

Maternal and Child Health; Nutritional Status; South American Indians

\section{Introdução}

Os indígenas Kaiowá e Guaraní sofreram, ao longo dos séculos, transformações sociais, econômicas e ambientais, que conduziram a mudanças negativas no seu viver e no adoecer. A perda da floresta enquanto espaço vital, a expulsão de suas terras, a degradação ambiental, a fome e a miséria em que vivem, atualmente, influenciam na sua saúde e nutrição 1,2 .

Os objetivos deste trabalho foram estudar o estado nutricional de crianças indígenas Kaiowá e Guaraní e conhecer as condições de saúde materno-infantil.

\section{População e métodos}

Os Guaraní contemporâneos são divididos em três subgrupos: Mbyá, Kaiowá e os Nhandéva/ Chiripá, sendo que apenas estes últimos se autodenominam Guaraní. No Estado de Mato Grosso do Sul, Brasil, estão dois destes subgrupos, os Kaiowá e os Guaraní. Vivem em 28 áreas indígenas da região sul do Estado e têm uma população de aproximadamente 30 mil indivíduos 1 .

Foi realizado um estudo transversal com uma amostra de 137 crianças indígenas Kaiowá e Guaraní com idade entre 0 e 59 meses, da Área Indígena de Caarapó, durante os meses de maio a setembro de 2003 . Nesse período, a po- 
pulação dessa área era de 2.800 indivíduos, sendo que 694 eram crianças com idade entre 0 e 59 meses. Todas as crianças estavam sendo acompanhadas pelo Programa de Vigilância Nutricional do Pólo Base de Caarapó. O Programa de Vigilância Nutricional avalia o estado nutricional por meio do índice peso/idade e utiliza a unidade de medida percentil, segundo as recomendações da Organização Mundial da Saúde (OMS) ${ }^{3}$. No período da pesquisa, o Programa de Vigilância Nutricional registrava $63 \%$ de crianças eutróficas (P10 a P97), 19\% sob risco nutricional (P3 a P10), 15\% desnutridas ( $\mathrm{P}<3$ ) e $3 \%$ obesas $(P>97)$. Respeitando essa proporcionalidade e utilizando-se um intervalo de confiança de $95 \%$, foram sorteadas 137 crianças. O sorteio priorizou o número de crianças e não o número de domicílios ou famílias, portanto, ocorreram casos em que mais de uma criança de uma mesma família participou da pesquisa.

O trabalho de campo consistiu em duas etapas: entrevista e avaliação nutricional, realizadas no domicílio das crianças e contou com a colaboração dos agentes indígenas de saúde (AIS) que facilitaram o diálogo e o aceite dos familiares.

Na entrevista foi aplicado um questionário, especificamente elaborado e que foi pré-testado, sobre as condições de saúde materno-infantil. A maioria das informações foi fornecida pelas mães. Apenas o registro de peso ao nascer foi verificado no cartão de nascimento ou de vacina da criança.

A antropometria foi baseada nas recomendações da OMS 3. As medidas obtidas foram peso e estatura, sendo registradas pela pesquisadora com a colaboração de dois AIS. As crianças foram pesadas em balança digital eletrônica marca Tanita, com precisão de $0,1 \mathrm{~kg}$. As crianças abaixo de $100 \mathrm{~cm}$ foram medidas em antropômetro de madeira tipo horizontal, e para crianças acima de $100 \mathrm{~cm}$ utilizou-se a fita métrica inelástica. Na impossibilidade de fixação da fita métrica na parede, utilizou-se o antropômetro digital eletrônico portátil da marca Soehnle.

O estado nutricional foi classificado pelos índices de peso/idade e altura/idade com base na unidade de medida escore-z: $<-2$ desnutridos, $-2 \mathrm{a}+2$ eutróficos $\mathrm{e}>+2$ obesos.

Para a análise estatística dos resultados foram utilizados os testes qui-quadrado e o exato de Fisher. O nível de significância utilizado foi de $\mathrm{p}<0,05$.

O projeto foi aprovado pelo Comitê de Ética em Pesquisa da Universidade Federal de Mato Grosso do Sul e pela Comissão Nacional de Ética em Pesquisa, contou também com a autorização das lideranças da Área Indígena de Caarapó.

\section{Resultados}

O número de crianças nas duas etapas do estudo foi diferente, visto serem realizadas em momentos distintos.

\section{Inquérito domiciliar $(n=137)$}

Das 137 crianças, 84 (61,3\%) eram meninas e $53(38,7 \%)$ meninos. Quanto à faixa etária, 15 crianças $(10,9 \%)$ tinham entre 0 e 5 meses, 20 $(14,6 \%)$ entre 6 e 11 meses, 39 (28,5\%) entre 12 e 23 meses e 63 (46\%) tinham entre 24 e 59 meses.

Nota-se que um número significativo das crianças tiveram baixo peso ao nascer $(<2.500 \mathrm{~g})$. Este número pode ser subestimado, visto a elevada ocorrência de crianças $(64,4 \%)$ que não tinham registro de peso ao nascer, sobretudo as nascidas nos domicílios e as que não tiveram acompanhamento pré-natal (Tabela 1).

\section{Avaliação nutricional $(\mathrm{n}=126)$}

$\mathrm{Na}$ antropometria houve perda de 11 crianças (8\%) por não terem sido encontradas no domicílio em até três tentativas. Das 126 crianças, 53 eram meninos e 73 meninas. Quanto à faixa

Tabela 1

Distribuição porcentual das características

da assistência pré-natal. Área Indígena

de Caarapó, Mato Grosso do Sul, Brasil, 2003

\begin{tabular}{lrr}
\hline Variáveis/categorias & $\mathbf{n}$ & $\%$ \\
\hline Peso ao nascimento (g) & & \\
$\quad$ < 2.500 & 14 & 30,4 \\
$>2.500$ & 32 & 69,6 \\
& & \\
Número de consultas pré-natal & & \\
Até 3 & 33 & 24,1 \\
4-6 & 67 & 48,9 \\
7 ou mais & 10 & 7,3 \\
Não realizou o pré-natal & 27 & 19,7 \\
& & \\
Local do parto & & \\
$\quad$ Domicílio & 73 & 53,3 \\
Hospital & 64 & 46,7
\end{tabular}


etária, $8(6,3 \%)$ tinham entre 0 e 5 meses, 12 (9,5\%) entre 6 e 11 meses, 37 (29,4\%) de 12 a 23 meses e $69(54,8 \%)$ de 24 a 59 meses. Na antropometria seis crianças, que na primeira etapa compuseram a amostra com idade entre $12 \mathrm{e}$ 23 meses, tinham completado 24 meses, aumentando a proporção de crianças da faixa etária de 24 a 59 meses.

Verificou-se uma elevada proporção de crianças com escore- $z<-2$. Além disso, observou-se uma marcada diferenciação entre o número de crianças desnutridas segundo o índice peso/idade e altura/idade, sendo que neste último a proporção foi mais elevada (Tabela 2).

A proporção de crianças desnutridas quando separadas por sexo, faixa etária e escolaridade materna não apresentou diferença estatisticamente significante para ambos os índices (Tabela 3).

\section{Discussão}

Um número expressivo de mães não teve atendimento pré-natal, os resultados obtidos foram superiores aos encontrados na Pesquisa Nacional de Demografia e Saúde (PNDS) na Região Centro-oeste, que foi de $7 \%$ e no Brasil de 13,2\% 4. A não realização do pré-natal pode aumentar o ris- co de morbidade e mortalidade materna e perinatal entre mães indígenas e seus filhos.

O trabalho desenvolvido pela equipe da Fundação Nacional de Saúde, com a imprescindível contribuição dos AIS, tem favorecido a maior adesão das gestantes ao acompanhamento pré-natal. Contudo, ainda existe a necessidade de estabelecer um melhor diálogo entre profissionais de saúde e mães, esclarecendo conceitos e conhecendo as práticas histórico-culturais desse povo.

Observou-se um número elevado de mães que fizeram seu parto no domicílio. Dado se-

\begin{tabular}{|c|c|c|c|c|}
\hline \multicolumn{5}{|c|}{$\begin{array}{l}\text { Distribuição das crianças indígenas segundo } \\
\text { os valores de escore-z para os índices peso/idade } \\
\text { e altura/idade, sexos combinados. Área Indígena } \\
\text { de Caarapó, Mato Grosso do Sul, Brasil, } 2003 .\end{array}$} \\
\hline \multirow[t]{2}{*}{ Escore-z } & \multicolumn{2}{|c|}{ Peso/idade } & \multicolumn{2}{|c|}{ Altura/idade } \\
\hline & $\mathrm{n}$ & $\%$ & $n$ & $\%$ \\
\hline$<-2$ & 23 & 18,2 & 43 & 34,1 \\
\hline$-2 a+2$ & 103 & 81,8 & 81 & 64,3 \\
\hline$>+2$ & - & - & 2 & 1,6 \\
\hline Total & 126 & 100,0 & 126 & 100,0 \\
\hline
\end{tabular}

Distribuição porcentual dos índices de altura/idade e peso/idade das crianças segundo o sexo, faixa etária e escolaridade materna. Área Indígena de Caarapó, Mato Grosso do Sul, Brasil, 2003.

\begin{tabular}{|c|c|c|c|c|c|c|c|c|c|c|c|c|}
\hline \multirow[t]{4}{*}{ Variáveis/categorias } & \multicolumn{12}{|c|}{ Escore-z } \\
\hline & \multicolumn{6}{|c|}{ Altura/idade (A/I) } & \multicolumn{6}{|c|}{ Peso/idade (P/I) } \\
\hline & \multicolumn{2}{|c|}{$<-2$} & \multicolumn{2}{|c|}{-2 a 2} & \multicolumn{2}{|c|}{$>+2$} & \multicolumn{2}{|c|}{$<-2$} & \multicolumn{2}{|c|}{-2 a 2} & \multicolumn{2}{|c|}{ Total } \\
\hline & $\mathrm{n}$ & $\%$ & $\mathrm{n}$ & $\%$ & $\mathrm{n}$ & $\%$ & $\mathrm{n}$ & $\%$ & $n$ & $\%$ & $\mathrm{n}$ & $\%$ \\
\hline \multicolumn{13}{|l|}{ Sexo* } \\
\hline Masculino & 21 & 39,6 & 30 & 56,6 & 2 & 3,8 & 12 & 22,7 & 41 & 77,3 & 53 & 100,0 \\
\hline Feminino & 22 & 30,1 & 51 & 69,9 & - & - & 11 & 15,0 & 62 & 85,0 & 73 & 100,0 \\
\hline \multicolumn{13}{|l|}{ Faixa etária (meses) ${ }^{\star \star}$} \\
\hline $0-5$ & - & - & 8 & 100,0 & - & - & - & - & 8 & 100,0 & 8 & 100,0 \\
\hline $6-11$ & 4 & 33,4 & 7 & 58,3 & 1 & 8,3 & 4 & 33,4 & 8 & 66,6 & 12 & 100,0 \\
\hline $12-23$ & 17 & 46,0 & 20 & 54,0 & - & - & 8 & 21,6 & 29 & 78,4 & 37 & 100,0 \\
\hline $24-59$ & 22 & 31,9 & 46 & 66,7 & 1 & 1,4 & 11 & 15,9 & 58 & 84,2 & 69 & 100,0 \\
\hline \multicolumn{13}{|l|}{$\begin{array}{l}\text { Escolaridade } \\
\text { materna (anos) }{ }^{\star \star \star}\end{array}$} \\
\hline Sem escolaridade & 18 & 38,3 & 28 & 59,6 & 1 & 2,1 & 11 & 23,4 & 36 & 76,6 & 47 & 100,0 \\
\hline $1-4$ & 19 & 32,8 & 38 & 65,5 & 1 & 1,7 & 8 & 13,8 & 50 & 86,2 & 58 & 100,0 \\
\hline $5-8$ & 6 & 28,6 & 15 & 71,4 & - & - & 4 & 19,1 & 17 & 80,9 & 21 & 100,0 \\
\hline
\end{tabular}

$\star p=0,103(A / I)$ e $p=0,351(P / I)$

$\star \star p=0,068(A / I)$ e $p=0,247(P / I)$.

$\star \star \star ~ p=0,904(A / I)$ e $p=0,445(P / I)$. 
melhante foi encontrado por Ribas et al. 5, sendo que $40 \%$ dos partos de mães indígenas Teréna de Mato Grosso do Sul foram domiciliares.

Constatou-se que as mães cujos filhos nasceram em épocas mais recentes receberam assistência pré-natal com maiores chances de cuidado hospitalar no parto. Contudo, as dificuldades de transporte e a demora no atendimento à gestante no momento do parto, constituem limitações que devem ser equacionadas para garantir um parto seguro e humanizado, seja este no ambiente hospitalar ou domiciliar. Quanto ao parto domiciliar, este deve ser incluído nas políticas de atenção à saúde, uma vez que nessa comunidade a parteira é respeitada e reconhecida, entretanto, este trabalho deve estar articulado à rede de serviços oferecidos à população.

Este estudo revelou um elevado número de crianças com baixo peso ao nascimento $(<2.500$ g), sendo superior ao obtido na PNDS que encontrou 9,1\% para a Região Centro-oeste e $8,1 \%$ no Brasil 4 . Ribas \& Philippi 6 observaram uma prevalência de $12,5 \%$ de baixo peso ao nascimento entre as crianças indígenas Teréna.

A ocorrência de desnutrição foi superior à encontrada por Ribas \& Philippi 6 entre os Teréna, que foi de $4 \%$ para o índice peso/idade e $11,1 \%$ de déficit estatural, e igual ou inferior quando comparada com povos indígenas de diferentes regiões brasileiras 7,8. Entre os Pakaanóva do Estado de Rondônia, a prevalência encontrada foi de $50 \%$ de déficit estatural e $30,8 \%$ de desnutrição por peso/idade entre as crianças de 24 a 59 meses de idade 8 .
A desnutrição foi maior no sexo masculino, comprovando o observado em outros estudos 6,9.

A partir do sexto mês de vida, todas as faixas etárias apresentaram déficits nutricionais. Deve-se destacar a influência das precárias condições sócio-econômicas, ambientais e de saúde desse povo podendo favorecer o aparecimento da desnutrição. Martins \& Menezes 9, investigando a evolução do estado nutricional de crianças menores de cinco anos das aldeias de Parakanã na Amazônia, descreveram a influência negativa do meio externo sobre o crescimento infantil, verificada pela redução nos valores dos índices antropométricos com o aumento da idade.

Observou-se maior número de crianças desnutridas entre as mães sem escolaridade, concordando com os estudos de Monteiro \& Freitas 10 que investigaram a influência da escolaridade materna sobre a saúde e nutrição infantil.

O presente estudo evidenciou elevada ocorrência de desnutrição e uma preocupante situação de saúde materno-infantil. A evolução e a expansão da oferta de serviços de saúde têm buscado alternativas para reverter esta situação. No entanto, estas ações ainda são incipientes, não chegando a prover melhorias das condições de saúde materno-infantil e de nutrição desse povo.

Destacamos a necessidade de maior número de pesquisas sobre a saúde materno-infantil e nutrição dos povos indígenas. Tais pesquisas devem fomentar e ampliar as discussões sobre as políticas e ações desenvolvidas pelos órgãos públicos, para reverter o perfil nutricional dos povos indígenas.

\section{Resumo}

Este trabalho teve como proposta estudar o estado nutricional de crianças indigenas e conhecer condições de saúde materno-infantil. Trata-se de um estudo transversal, com uma amostra de 137 crianças de zero a 59 meses de idade, das comunidades Kaiowá e Guaraní, Área Indígena de Caarapó, Mato Grosso do Sul, Brasil. Foram realizadas entrevistas com a aplicação de um questionário sobre as condições de saúde materno-infantil. A avaliação nutricional foi obtida por meio de medidas antropométricas (peso e estatura). Verificou-se que 19,7\% das mães não realizaram o prénatal e 53,3\% tiveram parto domiciliar. A ocorrência de desnutrição para o índice peso/idade foi de $18,2 \%$ e para o indice altura/idade foi de 34,1\%. A proporção de crianças desnutridas quando separadas por sexo, faixa etária e escolaridade materna não apresentou diferença estatisticamente significante para ambos os índices. Este estudo evidenciou elevada ocorrência de desnutrição infantil e uma preocupante situação de saúde materno-infantil.

Saúde Materno-Infantil; Estado Nutricional; Índios Sul-Americanos 


\section{Colaboradores}

R. P. Pícoli realizou o trabalho de campo e elaborou o artigo. L. Carandina e D. L. B. Ribas contribuíram na supervisão da análise dos dados e revisão crítica do artigo.

\section{Agradecimentos}

Aos agentes indígenas de saúde, em especial a Maria Celina e Neuza, pelo carinho e atenção dedicados a mim durante o trabalho de campo. À Fundação de Apoio à Pesquisa do Estado de São Paulo pelo auxílio pesquisa concedido (processo n. 03/05283-9).

\section{Referências}

1. Brand AJ. Desenvolvimento local em comunidades indígenas no Mato Grosso do Sul: a construção de alternativas. Interações - Revista Internacional de Desenvolvimento Local 2001; 2:59-68.

2. Grunberg FP. Reflexões sobre a situação dos Guarani no Mato Grosso do Sul, Brasil. Brasília: Equipe de Acompanhamento dos Guarani, Centro de Trabalho Indigenista; 2002.

3. World Health Organization. Physical status: the use and interpretation of anthropometry indicators of nutritional status. Geneva: World Health Organization; 1995. (Technical Report Series 854).

4. Victora CG, César JA. Saúde materno-infantil no Brasil - padrões de morbimortalidade e possíveis intervenções. In: Rouquayrol MZ, Almeida FN, organizadores. Epidemiologia \& saúde. Rio de Janeiro: Editora Medsi; 2003. p. 415-53.

5. Ribas DLB, Sganzerla A, Zorzatto JR, Philippi ST. Nutrição e saúde infantil em uma comunidade indígena Teréna, Mato Grosso do Sul, Brasil. Cad Saúde Pública 2001; 17:323-31.
6. Ribas DLB, Philippi ST. Aspectos alimentares e nutricionais de mães e crianças indígenas Teréna, Mato Grosso do Sul. In: Coimbra Jr. CEA, Santos RV, Escobar AL, organizadores. Epidemiologia e saúde dos povos indígenas no Brasil. Rio de Janeiro: Editora Fiocruz/ABRASCO; 2003. p. 73-88.

7. Coimbra Jr. CEA, Santos RV. Avaliação do estado nutricional num contexto de mudanças socioeconômicas: o grupo indígena Suruí do Estado de Rondônia, Brasil. Cad Saúde Pública 1991; 7:53862.

8. Escobar AL, Santos RV, Coimbra Jr. CEA. Avaliação nutricional de crianças indígenas Pakaanóva (Warí), Rondônia, Brasil. Rev Bras Saúde Mater Infant 2003; 4:457-61.

9. Monteiro CA, Freitas ICM. Evolução de condicionantes socioeconômicas da saúde na infância na cidade de São Paulo (1984-1996). Rev Saude Pública 2001; 34 (6 Suppl): 8-12.

10. Martins SJ, Menezes RC. Evolução do estado nutricional de menores de 5 anos em aldeias indígenas da Tribo Parakanã, na Amazônia Oriental Brasileira (1989-1991). Rev Saúde Pública 1994; 28:1-8.

Recebido em 23/Mar/2005

Versão final reapresentada em 11/Jul/2005

Aprovado em 27/Set/2005 